\title{
Feasibility study of non-contact measurement in mechanical verification method for dissolution instrument
}

\author{
Wei Zhou ${ }^{1, a^{*}}$, Mi Yao ${ }^{1, \mathrm{~b}}$ \\ ${ }^{1}$ Shanghai Institute Of Quality Inspection and Technical Research, Minhang, Shanghai, China
}

\begin{abstract}
Dissolution instrument is an effective alternative method to study and evaluate the bioavailability of drugs in vitro, and an important means to ensure and evaluate whether the production technology and quality of solid oral preparations are reasonable and stable. It is found that the mechanical properties of the instrument will affect the determination of the drug, which will lead to the deviation of the determination results. Some methods of mechanical verification of dissolution instrument are analyzed and the application of non-contact measurement method is explored in mechanical verification. Experimental results show that the proposed non-contact measurement method has good measurement accuracy and repeatability.
\end{abstract}

\section{Introduction}

The dissolution instrument replaces the previous disintegration time limit inspection with the scientific and advanced determination method, thus improving the scientific nature of the drug quality control method and ensuring the clinical effect of the drug[1]. But it is found that the mechanical properties of the instrument itself will affect the determination of drugs, including the stirring speed, the swing amplitude of the shaft, the vibration of the instrument, etc., which will lead to the deviation of the determination results[2,3]. The mechanical verification of the dissolution meter is the best method to reduce the influence of the variation factors and realize the dissolution result reproduction. Therefore, the pharmacopoeias of several countries have made detailed provisions on dissolution system in the appendix, and then supplemented the corresponding guidance on mechanical verification[4].

Since 1983, the US Food and Drug Administration (FDA) has carried out a lot of research work on the applicability of the dissolution system. In the early stage, the performance of dissolution is mainly verified by chemical correction sheet. Subsequently, the Pharmacopoeia Discussion Group (PDG), established by the United States Pharmacopoeia Commission, the European Pharmacopoeia Secretariat, and the Japanese Pharmacopoeia Commission, completed the coordination of the release and release test appendices in 2004. According to the European Pharmacopoeia and Japanese Pharmacopoeia, there are some defects in the correction sheet for calibration, and the only way to ensure the standardization of dissolution determination is to strictly check the geometric size of the instrument and the dynamic parameters of the dissolution instrument regularly[5].
In January 2010, the FDA's drug evaluation and research center published industry guidelines, which suggested mechanical calibration specifications available in the United States Pharmacopoeia (USP) dissolution instrument. It provided the guidance on "Mechanical Qualification of Dissolution Apparatus 1 and 2", to establish the standard process of the installation, mechanical calibration and operational inspection for the dissolution Apparatus 1(turn basket) and 2 (impeller), to ensure that the key parameters related to the dissolution conform to certain mechanical tolerances. The regulation requires mechanical calibration of the equipment during installation, after removal, and after repair. At the same time, the periodic calibration should be carried out once every 6 months[6].

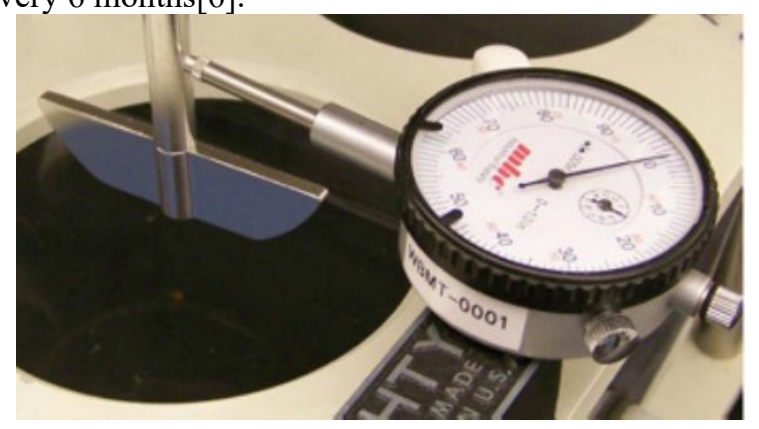

Fig. 1. dial meter

Among all the mechanical characteristics, the swing of the drive shaft is the most critical test item. In the Mechanical Verification Toolkit for Dissolution Instruments published by the United States Pharmacopoeia, the swing value is measured by a dial meter as shown in the Fig. 1. The American Agilent company has developed mechanical performance verification tool for its dissolution meter products specially. Although it is an integrated product, but for

\footnotetext{
${ }^{*}$ Corresponding author: a zhouwei2@sqi.org.cn

byaomi@sqi.org.cn
} 
the swing measurement is also used for contact measurement. However, because of the slight swing value, the contact measure method will lead to measuring error. A non-contact measurement method is proposed to detect the mechanical properties of the dissolution instrument.

\section{Non-contact measurement method}

Non-contact measurement is based on photoelectric, electromagnetic and other technologies, in the case of not touching the surface of the object to be measured, to get the surface parameter information of the measurement method. Typical non-contact measurement methods include laser method, eddy current method, ultrasonic measurement, machine vision measurement and so on. Considering the requirement and cost of measurement, the laser displacement sensor is chosen to carry out this work.

Laser displacement sensor uses laser technology to detect the position, displacement and other parameters of the object, which is generally composed of a laser, a laser detector and a measuring circuit. According to the measuring principle, the laser displacement sensor can be divided into two kinds: triangulation and echo analysis. The first method uses the laser scattered back from the surface of the measured object and receives it through the internal CCD linear camera. On the basis of different distances, the CCD linear camera observes the position of the reflected light point through imaging, and outputs different electrical signals according to the movement of the position. At present, the highest linearity of the laser displacement sensor using the triangulation method can reach $1 \mathrm{um}$, and the separation rate can reach the level of $0.1 \mathrm{um}$. The method of echo analysis calculates the distance by calculating the time it takes for the laser pulse to encounter the detector and return to the receiver, so it is also called the pulse-time method. The laser echo analysis method is suitable for long distance detection, but the measurement accuracy is lower than that of triangulation method, and the maximum detection distance can be up to $250 \mathrm{~m}$. In this paper, the displacement variation required to detect is close and the accuracy requirement is high, so the Keyence IL-30 laser sensor using triangulation method is selected. The Schematic diagram of triangular method is shown as Fig. 2.

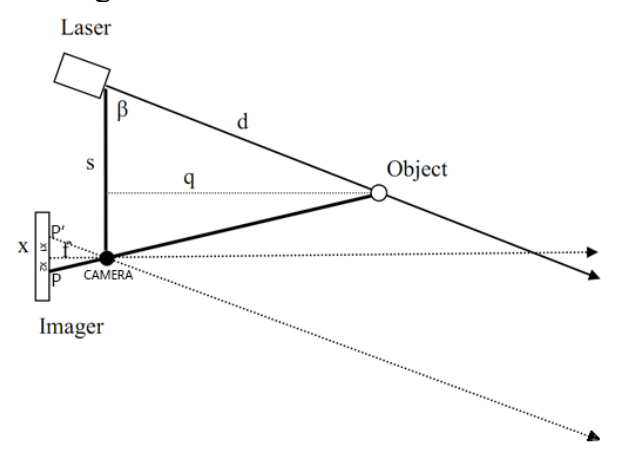

Fig. 2. Schematic diagram of triangular method
The calculation formula of $\mathrm{X}$ is

$$
X=x 1+x 2=\frac{f}{\tan \beta}+\text { psize } * \text { position }
$$

Where psize is the Pixel size, position is the pixel coordinate position of the image relative to the center of the image. Then the distance calculation formula are as follows:

$$
\begin{gathered}
q=f_{S} / X \\
d=q / \sin \beta
\end{gathered}
$$

Where $\mathrm{f}$ is the focal length of the camera, $\mathrm{s}$ is the distance between laser and camera and $\beta$ is the angle between the laser head and the reference line.

The swing value of the drive shaft can be directly measured by laser distance measuring directly without other device. Since the drive shaft rotates, the swing value at a certain point can reflect the degree of sloshing at that height. During a period of time when the dissolution meter is turned on and the laser senor is fixed, the difference between the maximum and minimum values of the displacement is the swing value.

\section{Measuring scheme design}

The software of the measuring scheme is designed in LabWindows/CVI which is an interactive $\mathrm{C}$ language development platform launched by National Instruments Corporation. The calculation program chart of swing value is shown in Fig. 3.

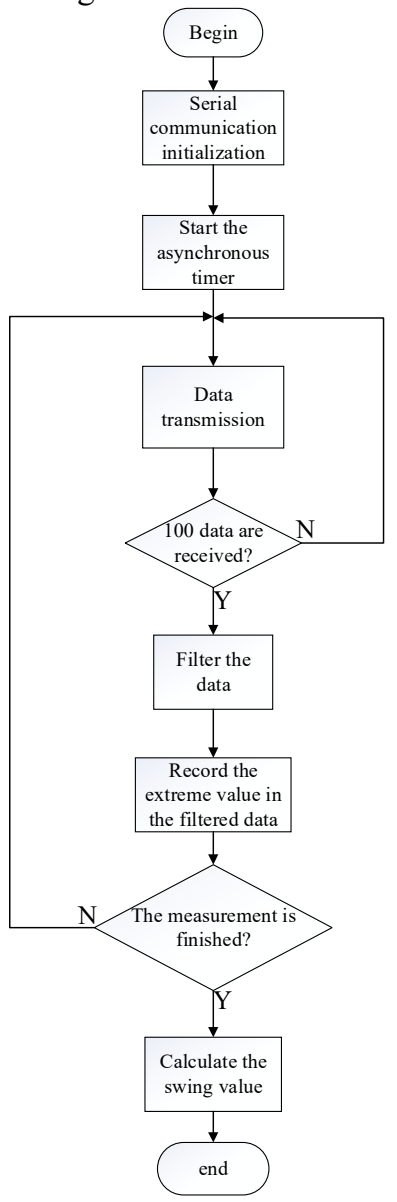

Fig. 3. calcaulation program chart 
Once the serial communication and the asynchronous timer is ready, the data of the laser sensor start transmission by the rate of 100 per second. Considering the calculation amount and the real-time performance, the data is processed every one second. In other words, once 100 hundred data are received, the data process is started. Since the measuring object is the stainless steel cylinder and the sampling rate is fast, the laser sensor occasionally picks up the wrong displacement. Therefore, the mean filtering algorithm is used to eliminate the outliers. After the data are filtered, the extreme values will be recorded until the measuring period is finished. The swing value can be directly obtained by the difference between the maximum and the minimum.

\section{Experiment and Result}

To verify the effectiveness of the non-contact measurement proposed for the detection of the swing value of the dissolution meter, the detection experiment was conducted. The experiment device includes a dissolution instrument, a laser pickoff unit, an $\mathrm{AD}$ conversion and the data analysis software, as shown in Fig. 4.

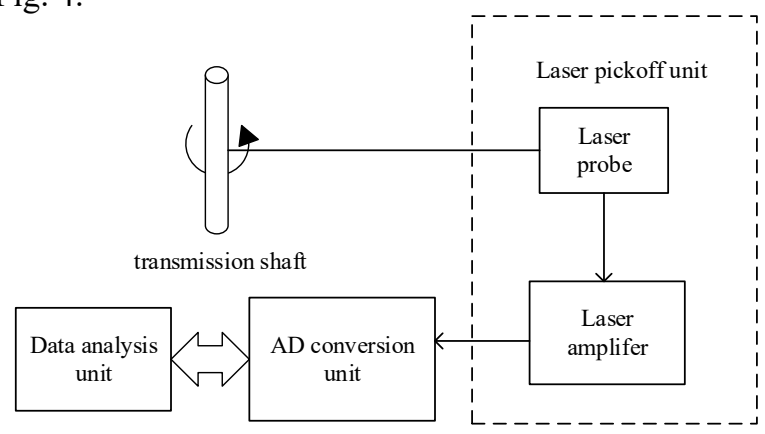

Fig. 4. Hardware connection diagram

At present, the high precision stripper brands on the market are all from developed countries, such as Hanson, Sotax, Agilent, Eweka, etc. In China, Tianda Tifa of Tianjin has designed and developed a dissolution meter with stable mechanical properties and easy operation in accordance with the requirements of the Chinese Pharmacopoeia and the United States Pharmacopoeia. In this paper, Tianda Tiafa RC80D was selected as the test object to verify its mechanical performance. As shown in Fig. 5, RC806D dissolution instrument adopts double row, eight glasses, eight pole, four pillars supporting structure form.

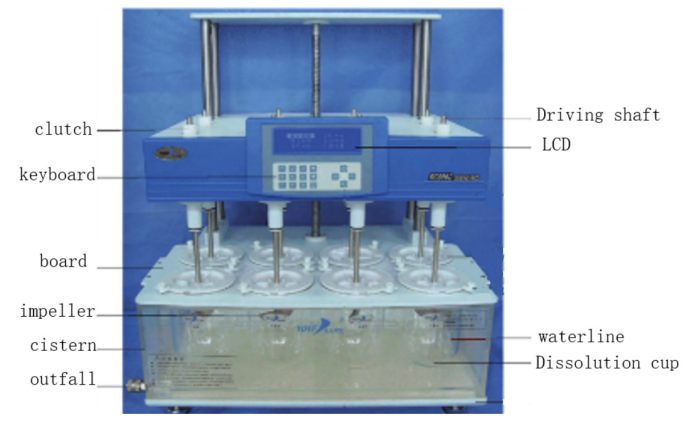

Fig. 5. RC80D dissolution instrument
In terms of laser sensor, Keyence IL-030 sensor unit working with IL-1000 amplifier unit was selected, their appearance is shown in Fig. 6. The body shell of IL-030 laser probe is made of PBT material and the metal part is made of SUS304 material, which has good environmental resistance. Its reference range is $30 \mathrm{~mm}$, the measurement range is $20 \sim 45 \mathrm{~mm}$, the red semiconductor laser source with $655 \mathrm{~nm}$ wavelength is adopted, the output power is $220 \mu \mathrm{W}$, and the minimum sampling period is $0.33 \mathrm{~ms}$. The minimum display unit of the IL-1000 amplifier is $1 \mu \mathrm{m}$. There are four analog output modes: $\pm 5 \mathrm{~V}, 1-5 \mathrm{~V}, 0-5 \mathrm{~V}$ analog voltage output and $4-20 \mathrm{~mA}$ analog current output. Its power supply voltage is $10-30 \mathrm{VDC}$, and the power consumption is up to $2300 \mathrm{~mW}$.

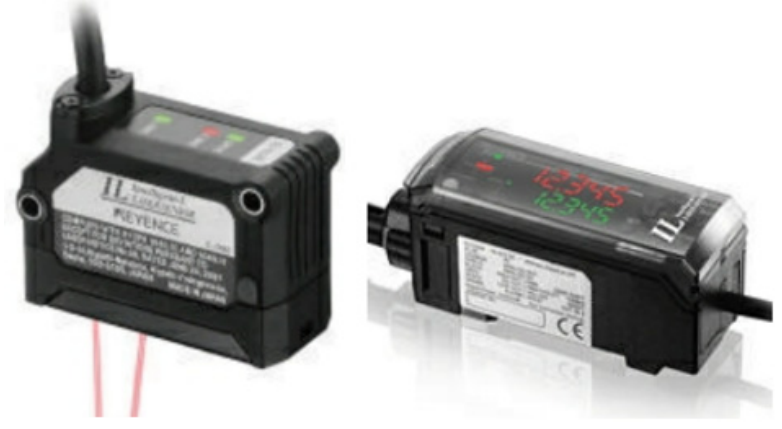

Fig. 6. laser probe and amplifier

The ADAM-4117 module of Advantech was selected as the data acquisition unit for the measuring system, which is a 16-bit A/D, 8-channel analog input module providing an independent programmable input range for every channel. The ASCII command was selected as the communication protocol and the sampling rate is set as 100 samples per second. The specific experimental steps and testing data are as follows.

Firstly, the laser probe is aligned with the center position of a certain height of the drive shaft, and the displacement of this center point is measured, as shown in the Fig. 7. Then, the dissolution meter is turned on and the data of the laser sensor is recorded for a period of time. By subtracting the minimum value from the maximum value, the maximum swing value of the drive shaft at this point can be measured during this period of time.

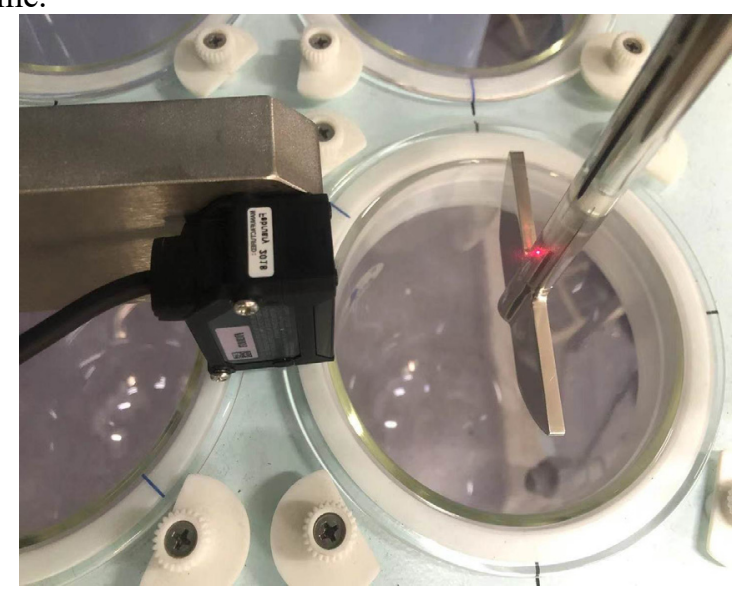

Fig. 7. measurement of swing value 
Table 1. measuring result of swing value

\begin{tabular}{|c|c|c|c|c|c|c|c|c|}
\hline & \multicolumn{7}{|c|}{ Swing value(mm) } \\
\hline $\begin{array}{c}\text { Dissolution cup } \\
\text { number }\end{array}$ & 1 & 2 & 3 & 4 & 5 & 6 & average & repeatability \\
\hline 1 & 0.100 & 0.088 & 0.088 & 0.095 & 0.105 & 0.095 & 0.095 & 0.0067 \\
\hline 2 & 0.125 & 0.145 & 0.131 & 0.130 & 0.135 & 0.135 & 0.134 & 0.0068 \\
\hline 3 & 0.160 & 0.165 & 0.162 & 0.135 & 0.160 & 0.165 & 0.158 & 0.0156 \\
\hline 4 & 0.180 & 0.165 & 0.168 & 0.175 & 0.160 & 0.165 & 0.169 & 0.0194 \\
\hline
\end{tabular}

Table 2. measuring result of swing value $5 \mathrm{~cm}$ above the impeller

\begin{tabular}{|c|c|c|c|c|c|c|c|c|}
\hline & \multicolumn{7}{|c|}{ Swing value(mm) } \\
\hline $\begin{array}{c}\text { Dissolution cup } \\
\text { number }\end{array}$ & 1 & 2 & 3 & 4 & 5 & 6 & average & repeatability \\
\hline 1 & 0.100 & 0.095 & 0.080 & 0.090 & 0.075 & 0.085 & 0.088 & 0.0088 \\
\hline 2 & 0.095 & 0.102 & 0.083 & 0.092 & 0.085 & 0.095 & 0.092 & 0.0076 \\
\hline 3 & 0.125 & 0.125 & 0.125 & 0.130 & 0.130 & 0.125 & 0.127 & 0.0026 \\
\hline 4 & 0.130 & 0.145 & 0.160 & 0.145 & 0.150 & 0.150 & 0.147 & 0.0098 \\
\hline
\end{tabular}

The four dissolution cups in front were selected as detection objects and two positions of the drive shaft were detected for every dissolution cup. The first position is $1 \mathrm{~cm}$ above the impeller and another is $5 \mathrm{~cm}$ above the impeller. The detection time is set as $15 \mathrm{~s}$ and every position was detected 6 times.

According to the testing data as shown in Table 1 and Table 2 , the repeatability is calculated by the formula as follows.

$$
\begin{gathered}
\bar{S}_{m}=\frac{1}{6} \sum_{n=1}^{6} S_{m n} \\
E_{r m}=\left[\frac{1}{5} \sum_{n=1}^{6}\left(S_{m n}-\bar{S}_{m}\right)^{2}\right]^{\frac{1}{2}}
\end{gathered}
$$

Where $S_{m n}$ are the actual testing value of the $\mathrm{nth}(\mathrm{n}=1,2,3,4,5,6)$ of the $\mathrm{mth}(\mathrm{m}=1,2,3,4)$ dissolution cup, $\bar{S}_{m}$ are the average swing value of the $\mathrm{mth}(\mathrm{m}=1,2,3,4)$ dissolution cup and $E_{r m}$ are the repeatability of the testing data of the $\mathrm{mth}(\mathrm{m}=1,2,3,4)$ dissolution cup.

By comparing the data in Table 1 and Table 2, the closer the measuring position is to the bottom of the drive shaft, the larger the swing value is, which is in line with its rotation physical characteristics. It can be seen from the test data that the repeatability of the test scheme reached $0.02 \mathrm{~mm}$.

\section{Conclusion}

A non-contacted measurement method is proposed in the mechanical verification of dissolution instrument to avoid the error caused by contacted measurement. The software of the measuring scheme is designed and a RC80D dissolution instrument was detected by a laser displacement sensor. The experiment results show that it is feasible to verify the mechanical properties of dissolution instrument and the repeatability of swing value is within $0.02 \mathrm{~mm}$.

\section{References}

1. Martin G P, Gray V A. Potential pitfalls when performing dissolution instrument qualification[J]. journal of gxp compliance, 2011.

2. Gregory, P., Martin, et al. Overview of dissolution instrument qualification, including common pitfalls[J]. Dissolution technologies, 2011, 18(2):6-10.

3. Gao Zongming, Moore Terry, Smith Anjanette P, et al. Gauge repeatability and reproducibility for accessing variability during dissolution testing: a technical note.. 2007, 8(4): E82.

4. Shuwen Zou, Jianguo Shan, Daojun Zhang.A Brief Discussion on Calibration Method of Stripper [J]. Equipment Manufacturing Technology, 2015(01): 239240.

5. Ge Lou, Haifeng Gu. Journal of Pharmaceutical Engineering, 2011, 18(24): 41-42.

6. YAN Quan, et al. Standard operating procedure for mechanical verification of drug dissolution meter [J]. Progress in Pharmacy, 2016, 40(09):702-7 